\title{
El impacto de la calidad educativa
}

\author{
The impact of educational quality
}

Hugo Bodero Delgado

Universidad Peruana de Ciencias Aplicadas

\section{INTRODUCCIÓN}

Hoy vivimos en una sociedad que en todas sus manifestaciones demanda calidad. Los países más desarrollados están experimentando un proceso de auge imparable en la valoración de la calidad. Su logro se constituye en una necesidad y a la vez en un problema ya que a pesar de su importancia crítica, permanece aún sin resolver en muchos lugares. Esto es uno de los grandes motivos para que la calidad de la educación sea un tema que en los últimos años haya concitado la atención de los especialistas.

Los contenidos que presentamos en este artículo giran en torno a la calidad de la educación. En la primera parte orientamos el artículo hacia una definición de calidad. Presentamos algunos argumentos sobre la necesidad de fijar el concepto de calidad; luego, un análisis de las diferentes maneras de conceptualizar este tema y las características más relevantes que tienen los diferentes conceptos de calidad educativa.

Propongo dar a conocer lo que se denomina calidad educativa porque este término ha tomado diversos conceptos en su significado, y solo en la medida en que se debata lo que ella implica y el alcance que adquiere, nos permitirá arribar a información resaltante y tratamientos adecuados.

\section{La complejidad de la calidad educativa}

La educación es un "sistema complejo", es decir, un sistema en el cual, en la totalidad o la

unidad, existe la diversidad, lo que significa que la unidad 0 totalidad es la síntesis de múltiples determinaciones. Un sistema complejo se caracteriza porque contiene múltiples subsistemas fuertemente conectados.

Calidad deriva del latín qualitas y según el diccionario de la Real Academia Española significa, propiedad $\circ$ conjunto de propiedades inherentes a una cosa

1 Licenciado en Psicología y estudios de posgrado en aspectos psicopedagógicos; docente de Posgrado de la Universidad Peruana de Ciencias Aplicadas (UPC), Lima. 
que permiten apreciarla como igual, mejor - peor que los restantes de su especie. El término en sí resulta polifacético y a menudo subjetivo y actualmente es uno de los conceptos más importantes en el mundo de las organizaciones, de ahí su importancia en procurar delimitarlo (1).

El concepto de calidad educativa: Es evidente que la educación no puede entenderse como un producto físico o manufacturado sino como un servicio que se presta a los alumnos. Pero, al igual que ocurre con otros servicios, la naturaleza de este servicio resulta difícil de describir, así como los métodos para evaluar la calidad (2).

La dificultad de definir la calidad educativa seguramente deriva de los siguientes hechos:

1. La educación es una realidad compleja en sí misma, ya que afecta a la totalidad del ser humano, entidad ciertamente compleja y multidimensional. Por ello, si resulta difícil precisar el resultado que se debe obtener de la educación, no debe extrañarnos que resulte complicado establecer métodos y criterios para determinar el nivel de calidad.

2. Existen notables diferencias entre las ideas o conceptos de lo que debe ser la educación. El resultado son las discrepancias sobre las metas o fines a lograr y sobre los procesos a realizar para lograrlo. Por ello, no disponemos de una teoría suficientemente consolidada para explicar la eficacia en el ámbito educativo.

3. Los procesos mentales de aprendizaje no son evidentes, y solo podemos inferirlos a través de los resultados que produce. En consecuencia, no podemos medir la actividad del intelecto de los alumnos, sino las manifestaciones externas de la actividad mental o intelectual.

4. El educador es un ser libre y el motivo último de su comportamiento es siempre su propia decisión, más allá de los modelos en los que se haya formado. Ello hace que la elección sobre el tipo de enseñanza o modelo educativo sea una elección personal, que no siempre se corresponde con la trayectoria o el ideario de la institución educativa.

La necesidad de explicar las diferentes dimensiones y los ejes fundamentales desde donde se puede reconocer la calidad de un sistema educativo, de una experiencia, o de una institución escolar, más que ser un problema teórico es parte de un ineludible compromiso profesional de poner a disposición de los tomadores de decisiones herramientas para facilitarles su tarea. Por esta razón, este artículo tiene como objetivo explicar con claridad la serie de opciones ideológicas y pedagógicas que enfrenta un tomador de decisiones cuando intenta mejorar la calidad de la educación. A partir de una detallada explicitación del concepto de calidad de la educación se examinan dos propósitos fundamentales: primero, para tomar decisiones que se orienten a mejorar la calidad de un sistema educativo concreto, y segundo, para realizar evaluaciones sobre una situación concreta que permite tomar decisiones para reorientar y reajustar procesos educacionales (3).

\section{Principios de la calidad educativa}

La calidad educativa cuenta con principios resaltantes como:

- La estructura del sistema educativo y la configuración y adaptación del currículo a las diversas aptitudes, intereses y expectativas de los alumnos.

- La función docente, garantizando las condiciones que permitan a los profesores el desarrollo de su labor, su formación inicial y permanente y su reconocimiento profesional.

- La evaluación del sistema educativo, de los centros y del rendimiento de los alumnos, de acuerdo con los estándares establecidos en los países del entorno europeo. 
- El fortalecimiento institucional de los centros educativos, mediante el refuerzo de su autonomía, la profesionalización de la dirección y un sistema de verificación de los procesos y los resultados.

- La determinación de las competencias y responsabilidades de los distintos sectores de la comunidad educativa, el clima de estudio y la convivencia en los centros escolares.

\section{Componentes de calidad en las instituciones educativas}

Si complejo resulta precisar el concepto de calidad educativa, mayor aún es la dificultad para precisar los niveles de calidad de la misma y determinar cuándo una institución educativa es de calidad.

Una primera aproximación es planteada por Gento Palacios cuando afirma que si "la finalidad esencial de las instituciones educativas parece ser el impulso y orientación de la educación en sus propios alumnos, podría considerarse que una institución educativa de calidad sería aquella en la que sus alumnos progresan educativamente al máximo de sus posibilidades y en las mejores condiciones posibles" (3).

Pero para sustentar esta afirmación necesitamos conocer una serie de aspectos del centro educativo relacionados con la organización, la gestión, la dirección, los resultados académicos, etc., y para ello precisamos disponer de unos referentes o componentes a los que con frecuencia se denomina variables.

Como señala Gento Palacios, a quien seguiremos en el desarrollo de este apartado, existen dos tipos de componentes: indicadores o identificadores y predictores (3).

Unos sirven para constatar la calidad como efecto, son los indicadores, que ponen de manifiesto el grado de calidad alcanzado y nos ofrecen el "perfil de calidad" de un centro educativo. Gento Palacios (3) los denomina variables dependientes o de criterio y la Fundación Europea para la Gestión de Calidad los llama resultados. Los componentes, indicadores o variables dependientes, de criterio o resultados se refieren a los componentes que permiten medir el grado de idoneidad de aquello que se evalúa, es decir, con la calidad de la institución (procesos, resultados, opinión de los clientes, etc.).

\section{Los identificadores de calidad}

Los identificadores 0 indicadores de calidad de una institución educativa son aquellos componentes que, relacionados con el producto o servicio conseguido, con la apreciación sobre el mismo y con los procesos de funcionamiento, permiten determinar la medida en que dicho centro educativo alcanza niveles de calidad en sus resultados.

Procesos + producto + apreciación

$=$ resultados

Los principales identificadores o indicadores de la calidad de un centro son: el producto educativo, la satisfacción de los alumnos, la satisfacción del personal que trabaja en el centro y el efecto de impacto de la educación alcanzada.

\section{El producto educativo como identificador de calidad}

La finalidad principal y última de un centro educativo es conseguir que sus alumnos alcancen niveles educativos de calidad. La calidad del centro, entendida en términos de resultados, estará íntimamente relacionada con la eficacia y sobre todo con la eficiencia en el aprovechamiento de los recursos y los procesos para la consecución de los objetivos educativos.

Según Gento Palacios (3), entre los criterios que podemos utilizar para medir el nivel de calidad del centro en relación con la calidad del producto están:

- Acomodación al grado de desarrollo de los alumnos (físico, intelectual, social y moral), a sus necesidades, intereses $y$ 
expectativas.

- Reconocimiento de los alumnos, padres, personal del centro y cuantas personas reciben el efecto o impacto del producto educativo.

- Permanencia o duración del producto o sus efectos en su ámbito social.

- Excelencia o perfección en relación con los fines o metas (objetivos del centro).

- Bajo costo de producción que no debe identificarse con presupuestos bajos, sino con el máximo aprovechamiento de los recursos.

- Disponibilidad o accesibilidad en el sentido de que el producto está tan extendido y es tan conocido y las vías para acceder a él son tan asequibles, que cualquier alumno pueda lograrlo.

- Cantidad de producción en relación con que lo alcancen o poseen un elevado número de alumnos (4).

El producto educativo típico de la institución educativa es la educación, como formación integral del ser humano, que se manifiesta en los valores. La cuestión siguiente es establecer qué valores han de conformar el producto educativo.

\section{El efecto de impacto de la educación como indicador de calidad}

El efecto de impacto se refiere a la repercusión que la educación de los alumnos que han pasado por el centro ha obtenido y su proyección en el entorno de trabajo donde desarrollan su actividad estas personas.

El efecto suele analizarse en cuatro ámbitos: académico, social, laboral y familiar.

- En el entorno académico se pone de manifiesto en tanto que una buena formación en una determinada etapa favorece el progreso en la siguiente.
- En el entorno familiar en tanto que influye en el clima familiar.

- En el entorno laboral si el alumno, en edad laboral, desempeña o simultánea el trabajo con el estudio o bien su situación laboral es buena por su buen nivel académico.

- En el entorno social en la medida en que las personas educadas en un centro ejercen una influencia sobre el entorno social en que se ubican: si el producto educativo es de calidad, se producirá un efecto favorable sobre dicho entorno: comportamiento cívico, clima de respeto y tolerancia social, colaboración y participación ciudadana, nivel cultural, mejora del medio ambiente, desarrollo de actividades físico-deportivas, etc.

El liderazgo es imprescindible para conseguir que el proyecto prospere. El liderazgo motiva a las personas, se inspira en convicción y entrega de los participantes, se desempeña como un arte y trata de convencer. Ahora bien, no debemos confundir liderazgo con dirección. La dirección sin liderazgo se sustenta en la legitimidad de la ley, se siente como un oficio e intenta ejercer el mando amparado por dicha legitimidad (4).

Los buenos líderes son los que trabajan con varios estilos en función de cada momento y situación. Cuantos más estilos practique el líder, mejor.

Un equipo directivo para ser eficaz, tiene que desarrollar cuatro tipos de actividades: directivas, administrativas, de innovación y de interacción.

La formación es un aspecto esencial de las estrategias de calidad pues incide directamente en el activo más valioso de un centro: en la "calidad" de su personal. La formación debe partir siempre de la situación en que se encuentra el profesor y del perfil que hay que conseguir, es decir, funciones que debe desarrollar y el 17 modelo de competencia profesional al que se aspira. El proceso de formación debe contemplar la reflexión y la solución de 
problemas.

El reconocimiento al esfuerzo y al trabajo bien hecho no solo es de justicia sino la palanca principal para motivar a las personas y mantenerlas ilusionadas con el proyecto. Este es, seguramente, uno de los aspectos menos aplicado en el sistema educativo y en la administración. Sin embargo, es un elemento esencial de cualquier plan de mejora.

La comunicación significa compartir y trabajar en equipo. Sin comunicación no hay participación, no hay implicación. Un aspecto esencial de la comunicación es la forma en que esta se produzca.

Aunque la mayoría de los centros educativos tiene conocimientos y recursos suficientes para elaborar su propio modelo de mejora, sin duda alguna es preferible la utilización de modelos contrastados y que permitan realizar comparaciones.

El mejorar la calidad educativa depende de que todos entendamos que es necesaria nuestra participación decidida y entusiasta y que no se requiere un cambio radical en nuestros sistemas de trabajo, sino más bien de un proceso de mejora continua, pero con un conocimiento y conciencia plena de lo que se quiere lograr. La calidad de la educación se resume en actitud y acción de mejora. Mejora que debe ser evidente a través de la medición.

Desde el punto de vista del docente, como educador y como profesional se traduce en un compromiso permanente en: cuidar los más mínimos detalles en la relación con la comunidad educativa, porque el nivel lo dan los pequeños detalles; formar, educar y proteger a los alumnos ofreciéndole toda las vías posibles para obtener su máxima educación; exigencia permanente de siempre ir a más; en ser constante en cuanto a las metas y flexibilidad en cuanto al método de trabajo (5).

Además de la preparación de los profesores, el éxito del alumnado depende de tres factores fundamentales: apoyo de la familia, dinámica en el aula y equipamiento de los centros.

Los factores asociados a la calidad educativa son múltiples y de diversa naturaleza. Están los factores de "insumo" (principalmente la formación del profesorado, de los alumnos y los materiales escolares), los factores de "contexto" (principalmente la escuela) y los factores de "respaldo" (principalmente la familia).

\section{CONCLUSIÓN}

La calidad educativa es una filosofía que, involucrando a toda la comunidad educativa, implica y compromete a todos en un proyecto común en el que se depositan toda expectativa de mejora y progreso. No debería ser asimilada a la eficiencia puesto que la calidad va más allá del rendimiento más o menos alto.

Definir cooperativamente y de manera colegiada el significado que queremos darle al termino calidad para las instituciones $y$ definir a los educadores.

Analizar cuáles son los programas convenientes para alcanzar, considerando el papel que deberán jugar los maestros en sus logros, y cuál deberá ser el proyecto de formación permanente para llegar al perfil ideal del maestro que se desea para una propuesta concreta de calidad.

\section{REFERENCIAS BIBLIOGRÁFICAS}

1. Instituto Peruano de Evaluación, Acreditación y Certificación de la Calidad de la Educación Básica. Equidad, acreditación y calidad educativa. Lima: Programa Educación Básica para Todos/IPEBA; 2011.

2. Organización de Estados Iberoamericanos para la Educación, Ciencia y Cultura. Una educación con calidad y equidad. España: OEl; 1998.

3. Gento S. Instituciones educativas para la calidad total. Madrid: La Muralla; 
1996.

4. Cano E. Evaluación de la calidad educativa. Madrid: La Muralla; 1998.

5. Piscoya L. Variables e indicadores para evaluar la calidad educativa. ¿QQuién dice qué es de calidad? XV Congreso Nacional de Educadores "Creando una Cultura de Calidad Educativa". Lima: Universidad Peruana de Ciencias Aplicadas; 2012. 